\title{
Exploiting multimode waveguides for pure fibre based fluorescence imaging
}

\author{
Tomáš Čižmár ${ }^{a}$ and Kishan Dholakia ${ }^{b}$ \\ ${ }^{a}$ School of Medicine, University of St. Andrews, North Haugh, St. Andrews KY16 9TF, UK; \\ ${ }^{b}$ SUPA, School of Physics and Astronomy, University of St. Andrews, North Haugh, St. \\ Andrews KY16 9SS, UK
}

\begin{abstract}
There has been an immense drive in modern microscopy towards miniaturisation and fibre based technology. This has been necessitated by the need to access hostile or difficult environments particulalrly in-situ and in-vivo. Strategies to date have included the use of specialist fibres and miniaturised scanning systems accompanied by ingenious microfabricated lenses. In parallel recent studies of randomized light fields and their holographic control opened up new ways for imaging. We present a novel approach for this field by utilising disordered light within a standard multimode optical fibre for minimally invasive lensless microscopy and optical mode conversion. We demonstrate scanning fluorescence microscopy at acquisition rates allowing observation of dynamic processes such as Brownian motion of mesoscopic particles. As the sample plane can be defined at any distance from the fibre facet, we eliminate the need for complex or elaborate focusing optics (e.g. miniaturized objectives, GRIN lenses) and instead reconfigure the system dynamically to image different axial planes. Furthermore, we show how such control can realise a new form of mode converter and generate various types of advanced light fields such as propagation-invariant beams and optical vortices. These may be useful for future fibre based implementations of super-resolution or light sheet microscopy. To the best of our knowledge, this technology represents the narrowest possible image guiding system based on light propagation.
\end{abstract}

Keywords: Digital holography, multimode waveguides, fluorescence, microscopy

\section{INTRODUCTION}

Complete control over the phase, amplitude and polarisation of a light field may lead to startling results. In the last few years we have seen the onset of revolution in this area with particular regard to random media. ${ }^{1-3}$ By detailed analysis of the output light we can adjust the input wavefront of a given light beam to control light propogation to unprecedented depths and to focus light at a given point in space despite deleterious scattering and the presence of severe aberrations. At the heart of this method is the realisation that the object has a form of a transmission matrix that can be determined and utilized by feedback to a dynamic diffractive optical element to shape the incident wavefront for enhanced transmission and beam focusing. This area has already led to a new understanding of light propagation and the preliminary applications of imaging and micromanipulation at depth in free space optical systems. The concept of disorder is not restricted to such randomised media. In fact we may consider various photonics structures in the same regard, particularly those that have the ability to simultaneously support multiple modes of optical propagation. Such multimode waveguides are ubiquitous in numerous photonics experiments but are typically limited by modal dispersion restricting their application. Such dispersive effects lead typically to various forms of modal interference and 'unpredictable' output beam profiles such a speckle patterns, depending critically upon the interaction of these modes as they propagate along the length of a waveguide.

Separately we are seeing a burgeoning need for imaging devices that offer observation in areas with typically very limited access. In the biomedical arena, optical endoscopes aim to fulfil this need. In tandem with advanced forms of microscopy and optical sectioning, remarkable progress has been made in the miniaturization and resolution enhancement of imaging modalities. Endoscopes have been developed that deliver in vivo fluorescence,

Further author information: (Send correspondence to T.C.)

T.C.: E-mail: tc51@st-andrews.ac.uk

Advanced Biomedical and Clinical Diagnostic Systems XI, edited by Anita Mahadevan-Jansen,

Tuan Vo-Dinh, Warren S. Grundfest, Proc. of SPIE Vol. 8572, 85720S · C 2013 SPIE

CCC code: $1605-7422 / 13 / \$ 18 \cdot$ doi: $10.1117 / 12.2003798$

Proc. of SPIE Vol. 8572 85720S-1 
confocal and mulitphoton microscopy and have yielded new insights into molecular, cell and tissue biology. ${ }^{4}$ For sixty years, the majority of endoscopes have relied on image transfer through optical fibre bundles. This technology heralded a major improvement in endoscopic imaging as within a flexible instrument ${ }^{5,6}$ yet does pose numerous challenges in design and implementation. Modern compact endoscopes (fiberscopes), particularly those used for in-vivo studies, typically focus light through a miniaturized objective ${ }^{7}$ or a gradient-index (GRIN) lens ${ }^{8}$ that creates an image on an input facet of a coherent fibre bundle which acts as an image guide. In most of the cases anterior imaging optics dictates the final dimensions of the instrument: The diameter of a miniaturized objective is currently typically a few millimeters, and around a millimeter for the GRIN lens. Using endoscopes without such optics is possible ${ }^{9,10}$ however, it restricts the imaging plane to the fiber facet.

In this article, we exploit our understanding of light transmission through disorder to show image information does not need to be separated into such individual channels in-situ as might be construed from the present understanding of endoscopy, but show that the whole image in the field of view can be transmitted simultaneously within a single-core multimode waveguide and reconstructed ex-situ behind the fiber exploiting the principles of digital holography. The resolution of this multimode 'fibrescope' is given by the numerical aperture (NA) of the used fibre unlike for the case of fibre bundles, where the imaging resolution is given by the anterior optics and the spatial separation of individual channels. Importantly, our method allows us to record images at any arbitrary axial distance behind the fibre facet. As we increase the distance from the fibre facet, a trade-off occurs: a reduction of spatial resolution occurs but we obtain a wider field of view. As the total number of modes allowed to propagate in the fibre is fixed, the final information content of the image remains unchanged regardless of the axial distance where the system operates. Our 'disorder' based fibrescope cross-section is dictated purely by the size of the multimode fibre itself.

A major advantage of considering and exploiting a multimode fibre as a disordered medium is that the equivalent image information can be relayed through a much narrower cross-section than is possible with fibre bundles. For example in a commonly used fibre bundle, Sumitomo IGN-08/30, ${ }^{10,11} 30,000$ guiding cores are contained within an area of $720 \mu \mathrm{m}$ in diameter. At the wavelengths of $0.5 \mu \mathrm{m}$ and for the the same NA of 0.35 , a multimode fibre, with diameter of about $140 \mu \mathrm{m}$, allows one to transfer the same amount of image information as it allows accordant number of modes with one polarization to propagate through. Even if the size of the guiding channels is reduced to the limiting case of a single mode waveguide with NA as high as $\approx 1,{ }^{12}$ a multimode fibre constructed from the same materials and diameter would still transfer approximately an order of magnitude higher number of modes. Scanning a single-mode fibre across the imaging plane would lead to a similar resolution with even smaller diameter fibre optics, however, that would require additional anterior optics and substantial mechanical actuators for its functionality giving the instrument a dimension of the order of one millimeter. ${ }^{13,14}$ Therefore we contend that our methodology paves the way for inexpensive lensless endoscopes with diffraction limited resolution and flexibility in imaging depths. This may open up now routes for advanced surgery and imaging within scenarios with difficult access such as turbid biological environments.

The results we present put forward several approaches to exploit multimode waveguides for imaging. We demonstrate a geometry for fluorescent imaging where excitation wavelength is delivered in situ by the same waveguide that also collects the fluorescence signal. Furthermore, we also present an approach for using the very same fibre as a mode converter, providing various complex beam types behind the fiber. This could pave the way for new fibre based versions of super-resolution imaging or light-sheet microscopy. At the present stage of the development the fibre has to be kept static after its calibration for the duration of the experiment This limits the spectrum of applications to rigid endoscopes. It is crucial to note, we can approach video-rate imaging for a very limited image resolution, however, the system performance can be significantly improved even with better use of currently available technology.

\section{RESULTS}

\subsection{Scanning fluorescent imaging}

In the heart of the technique is a method to acquire complex knowledge about light propagating through the multimode fibre (transformation matrix). Such procedure taking into account all the necessary aspects of the light field - phase, amplitude and polarisation was recently demonstrated and applied for the purpose of micromanipulation ${ }^{15}$ and will be henceforth referred to as "the calibration". 
When fluorescent imaging is required, the fluorescence emission band and the excitation wavelengths are very distinct from each other and any calibration performed would not be valid for both of them simultaneously. In fact, the fluorescence signal is typically emitted with a very broad spectrum that could not be subsequently analyzed behind the fiber without major power losses while filtering out a single wavelength. On the other hand the excitation wavelengths can be used in a very narrow bandwidth. Using the knowledge of the fiber field transformation they can be delivered through the fiber sequentially into distinct points across the sample plane (sample modes). The fluorescent signal may subsequently be collected throughout the fibre and recorded by an appropriate detector. Reconfiguring the SLM for measurement of each of the sample point sequentially is straightforward but even with the fast modulator we use in our geometry (Boulder Nonlinear Systems) we only reach rates of the order of $100 \mathrm{~Hz}$. An image containing 1000 data-points would thus be scanned in the relatively long interval of 10s. This however compares very favourably with a related approach recently published, ${ }^{16}$ where an image of $60 \times 60$ points was obtained within an interval of 3 minutes.

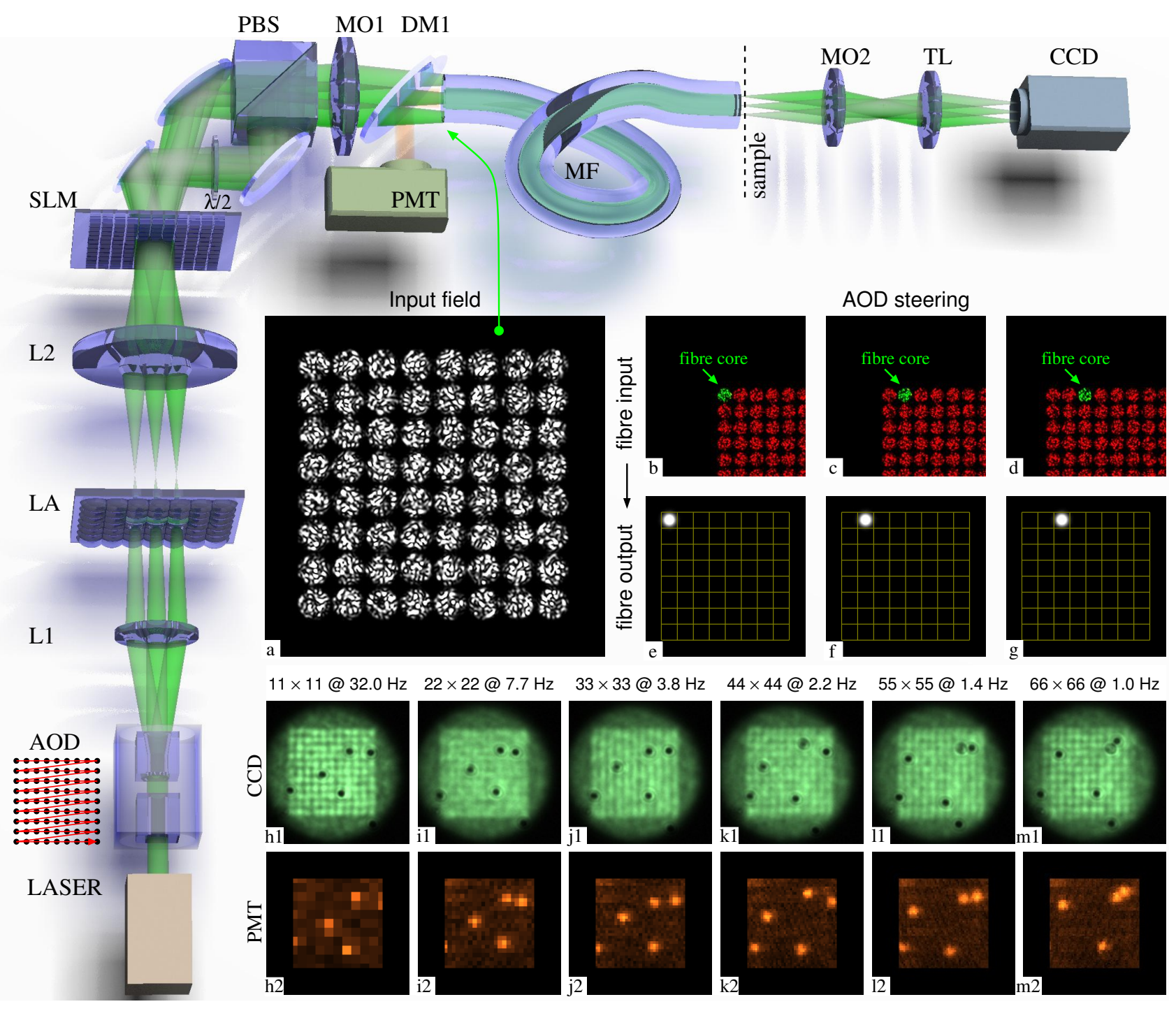

Figure 1. The experimental geometry, principles of operation and sample images for the multimode fibre based fluorescence microscopy. a, SLM generated matrix of optical fields corresponding to a series of sample modes - tightly focused beams at the sample plane. b-d, The AOD sequentially aligns individual segments on the fiber core, that results in scanning of focussed beam along the sample plane (e-g). h-m, Fluorescent imaging of fluorescent polymer particles having $2 \mu \mathrm{m}$ in diameter at various resolutions and frame-rates. 
In contrast to the SLM, the technology of the AOD can be employed to redirect a laser beam with very fast rates, but does not lend itself to more complex beam-shaping due to the absence of facile phase shaping. Both technologies were developed in parallel in the branch of optical manipulation, but only recently, they were combined into more powerful tandem system that immediately manifested itself as an extraordinary optical tool allowing fast and complex beam-shaping. ${ }^{17,18}$ We now show how to utilize this assembly to enhance the speed of the fibre-based fluorescent imaging by two orders of magnitude.

Our geometry is presented in the fig. 1. To excite red fluorescence dye, we built the system using the wavelength of $532 \mathrm{~nm}$. After the calibration procedure, we generate an imaging hologram as a complex superposition of modulations corresponding to a single sample mode, each amended by specific phase tilt. If applied on the uniformly illuminated SLM, the resulting light distribution at the input fiber facet forms a matrix of non-overlapping optical landscapes - generating fields - each confined within an area corresponding to the size of the fibre core (fig. 1 a). Each of these fields generates a specific sample mode if coupled into the fiber (fig $1 \mathrm{~b}-\mathrm{g}$ ). During the imaging process, individual field regions are sequentially aligned onto the fibre core by steering the laser beam with the AOD. The range of deflection angles the AOD can provide, however does not fully cover our requirements. Since for this application we do not need the full spectrum of the deflection angles, only a series of discrete values, we can efficiently magnify the deflection range combining the AOD with a lenslet array (LA). This way we can steer the focussed beam within the sample at very high rates constrained only by the AOD rise/fall times. The fluorescence signal is registered at a photomultiplier PMT for every AOD configuration at rates of $40 \mathrm{kHz}$. Resulting pixel resolution of $11 \times 11$ image points do not provide sufficient sampling of the fibre output area. To enhance the resolution we synthesize a number of different imaging holograms to be sequentially exposed by the SLM, each illuminating different array of $11 \times 11$ image points. Resulting image is generated combining detected fluorescence values obtained for all of the imaging holograms. Due to a number of synchronization issues between the controlling computer, SLM, AOD and PMT, we could only operate the system at about half of the rate corresponding to the slowest component (SLM). The performance of the fluorescent imaging is presented in fig. $1 \mathrm{~h}-\mathrm{m}$ for varying resolutions and corresponding frame-rates.

\subsection{Advanced beam-shaping for complex imaging methods}

As we now progress to show, exploiting and controlling the disorder in multimode fibers may yield a number of non-zero order modes that are essential for more complex imaging geometries including super-resolution techniques. The multimode fibre in this instance acts akin to a mode converter. As discussed for example in, ${ }^{19}$ the light propagating within the fiber is not randomized with loss of all parameter knowledge. A very important aspect in the randomization process is utiliised: light coupled into the fiber with specific propagation constant $k_{z}$ (axial component of the $\mathbf{k}$ vector), will leave the fiber output with this quantity conserved (see fig. 2 a). The coupling between radial modes is described by a coupling coefficient, that depends on the fibre quality and lengths, but typically is very low. Hence in our geometry, modes located within a certain annular zone at the SLM plane will form an orthogonal sub-space not interacting (significantly) with modes at other zones. This means that any azimuthally independent spectral modulation of the optical field at the fibre input will have identical influence on the spatial spectrum of the output field. In other words, for azimuthally independent spectral modulations, the multimode fibre behaves as a Fourier component. This substantially broadens the spectrum of light shapes that we can generate in the sample: Adding an appropriately sized piston with phase elevation of $\pi$ (fig. $2 \mathrm{~b}$ ) to the original modulation, the initial ideally focussed sample mode gets converted to a 'bottle' beam. ${ }^{20}$ Its lateral and longitudinal cross-sections are presented in fig. 2 e,f. This might for example allow performing stimulated emission depletion (STED) microscopy deep within turbid media employing fiber based system operating at two wavelengths. Similarly, annular filtering (fig. 2 c) transforms the sample mode to a zero-order Bessel beam (fig. $2 \mathrm{~g}, \mathrm{~h}$ ) that can be used for a broad spectrum of optical manipulation techniques, and if rapidly steered along one direction (using a tandem SLM-AOD system), it will generate an ideal field for light-sheet microscopy (LSM) ${ }^{21}$ This may be delivered within turbid environments by the fibre directly to the imaging plane without scattering losses and randomization. A further very important demonstration is that of a quadratic radial modulation $(2 \mathrm{~d}$ ), that causes an axial shift of the sample mode (or any other beam type). This way one can refocus upon another axial plane when imaging without need to recalibrate the system. Longitudinal cross-sections of a sample mode axially positioned into several different sample depths are shown in fig. 2 i. Other applications of this special feature might involve manipulation of NA of sample modes (that we 
employed for fluorescent imaging) or in combination with appropriate illumination, fiber based phase-contrast microscopy.

The versatility of the method is seen from the fact that any other beam shape required for a specific application can be formed by combining sample modes with an appropriate amplitude, phase and polarization. ${ }^{15}$ A key illustrative example is the generation of optical vortices, that we create from a number of modes positioned along a circle of an adequate radius (with the radial index of $p=0$ ) and gradually modify the phase of the sample modes accordingly to the required topological charge (integer number of $2 \pi$ phase elevations along the circumference, see fig. $2 \mathrm{j}$ ). A sequence of optical vortices with topological charge from an interval of $l \in<-3,3\rangle$ is presented in fig. $2 \mathrm{k}$, together with interferograms of vortices of $l= \pm 1$ with a plane-wave while gradually increasing a phase delay between them, thus manifesting the helicity of the resulting wavefront, typical for these fields (fig. $2 \mathrm{l})$.

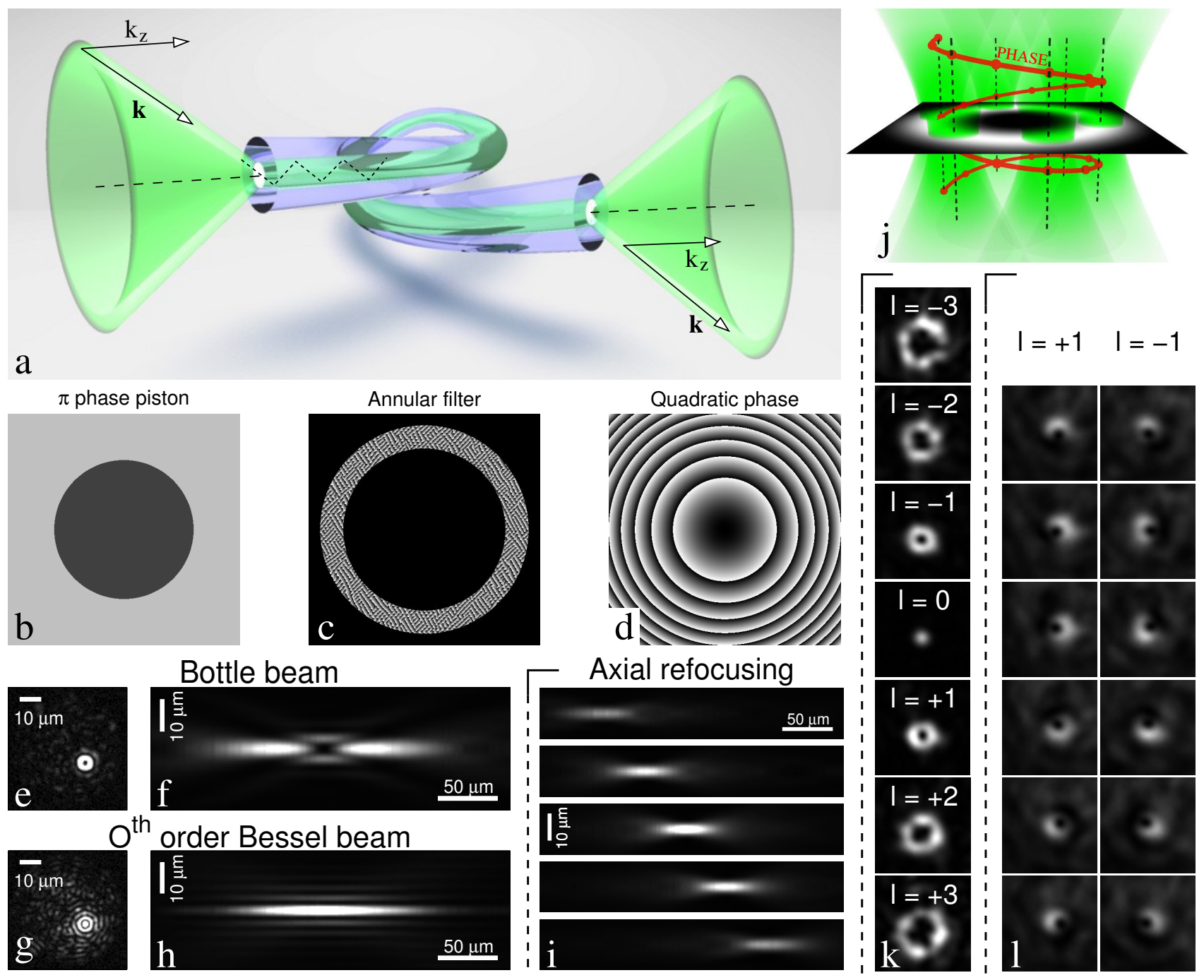

Figure 2. Shaping of output modes. a, conservation of propagation constant. b-d, azimuthally independent modulations leading to generation of bottle beam $(e, f)$, zero order Bessel beam $(g, h)$ and axial positioning of an output mode (i). $\mathrm{j}$, generation of optical vortices from a series of fundamental output modes. $\mathrm{k}$, experimentally generated optical vortices with varying topological charge. 1 , interferograms of $l= \pm 1$ vortices with a plane wave for 6 different phase shifts of the plane wave covering an interval of $\langle 0,2 \pi\rangle$. 


\section{DISCUSSION}

Controlled light propagation through disorder opens up new forms of imaging. In particular the use of a simple step-index multimode optical fibre shows startling new ways to acquire images as well as form a range of non zero order light beams. Coherent light propagating through such waveguide is randomized but the image information is not lost and can be decoded once the overall system transformation is measured. As the sample plane can be defined at any distance from the fibre facet, we can eliminate the need for complex or elaborate focusing optics (eg miniaturized objectives, GRIN lenses) and reconfigure the system dynamically at the SLM to image different axial planes. Our experiments were performed in the vicinity of the fiber to achieve the highest spatial resolution, but one can select another remote plane to gain an increased field of view. This allows a 'zooming' ability of the system providing multiple calibrations at a series of different axial sample planes. Using multimode fibers also allows a eduction in the size of the image guide by an order of magnitude compared to currently available fibre bundles.

We have presented an experimental geometry capable of forming a rapidly scanning illumination beam into the sample through the multimode fibre core. This simultaneously collects and delivers (backwards) the fluorescent signal to be detected on the fibre output.

The instrument was successfully tested for the imaging of polymer particles in close proximity of the fibre facet, with sufficient resolution for sampling of the fibre core area and an imaging speed approaching videorates. The geometry was built with standard commercially available components, leading to a relatively limited frame-rate and resolution, that could be vastly improved in the future.

To the best of our knowledge, this technology could represent the narrowest possible image guiding system based on light propagation. The spectrum of applications is very broad, with particular importance for minimally invasive in-vivo endoscopy allowing collateral damage in the observed substance to be kept as low as possible.

Future work will explore improvements in resolution and imaging rates, but also the employment of other waveguide types, especially gradient-index multimode optical fibres, that could minimize requirements for a high spectral purity of the used laser source and possibly allow multi-photon excitation. We will explore application of this technology for other advanced types of microscopy (STED, LSM) and continue fundamental studies of the light randomization process within the fibre, that might allow additional flexibility of the instrument during imaging. More details about these approaches can be found in ${ }^{22}$

\section{ACKNOWLEDGMENTS}

T. C. acknowledges support from the University of St Andrews. K. D. acknowledges support from EPSRC. K. D. is a Royal Society-Wolfson Merit Award holder.

\section{REFERENCES}

[1] Vellekoop, I. M. and Mosk, A. P., "Focusing coherent light through opaque strongly scattering media," Opt. Lett. 32, 2309-2311 (AUG 15 2007).

[2] Čižmár, T., Mazilu, M., and Dholakia, K., "In situ wavefront correction and its application to micromanipulation," Nat Photon 4, 388-394 (06 2010).

[3] Popoff, S. M., Lerosey, G., Carminati, R., Fink, M., Boccara, A. C., and Gigan, S., "Measuring the transmission matrix in optics: An approach to the study and control of light propagation in disordered media," Phys. Rev. Lett. 104, 100601 (Mar 2010).

[4] Flusberg, B. A., Cocker, E. D., Piyawattanametha, W., Jung, J. C., Cheung, E. L. M., and Schnitzer, M. J., "Fiber-optic fluorescence imaging," Nat Meth 2, 941-950 (12 2005).

[5] Hopkins, H. H. and Kapany, N. S., "A flexible fibrescope, using static scanning," Nature 173, 39-41 (01 1954).

[6] Berci, G., "Professor Harold H. Hopkins," Surgical Endoscopy 9, 667-668 (1995). 10.1007/BF00187935.

[7] Sung, K.-B., Liang, C., Descour, M., Collier, T., Follen, M., and Richards-Kortum, R., "Fiber-optic confocal reflectance microscope with miniature objective for in vivo imaging of human tissues," Biomedical Engineering, IEEE Transactions on 49, 1168 -1172 (oct. 2002). 
[8] Flusberg, B. A., Nimmerjahn, A., Cocker, E. D., Mukamel, E. A., Barretto, R. P. J., Ko, T. H., Burns, L. D., Jung, J. C., and Schnitzer, M. J., "High-speed, miniaturized fluorescence microscopy in freely moving mice," Nat Meth 5, 935-938 (11 2008).

[9] Sun, J., Shu, C., Appiah, B., and Drezek, R., "Needle-compatible single fiber bundle image guide reflectance endoscope," Journal of Biomedical Optics 15(4), 040502 (2010).

[10] Muldoon, T. J., Pierce, M. C., Nida, D. L., Williams, M. D., Gillenwater, A., and Richards-Kortum, R., "Subcellular-resolution molecular imaging within living tissue by fiber microendoscopy," Opt. Express 15, 16413-16423 (Dec 2007).

[11] Lane, P. M., Dlugan, A. L. P., Richards-Kortum, R., and MacAulay, C. E., "Fiber-optic confocal microscopy using a spatial light modulator," Opt. Lett. 25, 1780-1782 (Dec 2000).

[12] Ghaemi, H. F., Li, Y., Thio, T., and Wang, T., "Fiber image guide with subwavelength resolution," Applied Physics Letters 72(10), 1137-1139 (1998).

[13] Myaing, M. T., MacDonald, D. J., and Li, X., "Fiber-optic scanning two-photon fluorescence endoscope," Opt. Lett. 31, 1076-1078 (Apr 2006).

[14] Engelbrecht, C. J., Johnston, R. S., Seibel, E. J., and Helmchen, F., "Ultra-compact fiber-optic two-photon microscope for functional fluorescence imaging in vivo," Opt. Express 16, 5556-5564 (Apr 2008).

[15] Čižmár, T. and Dholakia, K., "Shaping the light transmission through a multimode optical fibre: complex transformation analysis and applications in biophotonics," Opt. Express 19, 18871-18884 (Sep 2011).

[16] Bianchi, S. and Di Leonardo, R., "A multi-mode fiber probe for holographic micromanipulation and microscopy," Lab Chip ,- (2012).

[17] G.M. Akselrod, Timp, W., Mirsaidov, U., Zhao, Q., Li, C., Timp, R., Timp, K., Matsudaira, P., and Timp, G., "Laser-guided assembly of heterotypic three-dimensional living cell microarrays," Biophys. J. 91, 3465-73 (2006).

[18] Čižmár, T., Dalgarno, H. I. C., Ashok, P. C., Gunn-Moore, F. J., and Dholakia, K., "Interference-free superposition of nonzero order light modes: Functionalized optical landscapes," Applied Physics Letters 98(8), 081114 (2011).

[19] Gambling, W. A., Payne, D. N., and Matsumura, H., "Mode conversion coefficients in optical fibers," Appl. Opt. 14, 1538-1542 (Jul 1975).

[20] Arlt, J. and Padgett, M. J., "Generation of a beam with a dark focus surrounded by regions of higher intensity: the optical bottle beam," Opt. Lett. 25, 191-193 (Feb 2000).

[21] Huisken, J., Swoger, J., Del Bene, F., Wittbrodt, J., and Stelzer, E. H. K., "Optical sectioning deep inside live embryos by selective plane illumination microscopy," Science 305(5686), 1007-1009 (2004).

[22] Čižmár, T. and Dholakia, K., "Exploiting multimode waveguides for pure fibre-based imaging," Nat Commun 3(1027) (2012). 